\title{
Fabrication of Substitution-Box Initiated Implementing Invertible Mapping and Improving its Competency of Confusion by Compliment's Mechanism
}

\author{
Muhammad Sarfraz \\ School of Mathematics \\ Sun Yat-sen University \\ China \\ msarfrazmphil@gmail.com
}

\author{
Yongjin Li \\ School of Mathematics \\ Sun Yat-sen University \\ China \\ stslyj@mail.sysu.edu.cn
}

Fateh Ali

School of Mathematics \&Statistics

Xi’an Jiaotong University

China

fatehalirana47@gmail.com

\author{
Zain-Ul-Abideen Haroon \\ Department of Computer Science \\ Virtual University, Pakistan \\ sardarzain29@yahoo.com
}

\author{
Akhter Rasheed \\ Department of Mathematics \\ CIIT, Abbottabad, Pakistan \\ akhter@ciit.net.pk
}

Recived : 8\1\2018

Revised : 7\2\2018
Accepted : $12 \backslash 2 \backslash 2018$

Available online : $\quad 20 / 2 / 2018$

DOI: $10.29304 / j q c m .2018 .10 .2 .367$

\section{Abstract}

In this research article, an innovative strategy is exploited to design a nonlinear component Substitution Box (S-box). To achieve an objective, initially we pick out a one specific sort of primitive irreducible polynomial of degree 8 to generate elements of Galois field $G F\left(2^{8}\right)$. Furthermore, we established a precise category of invertible mapping through an employment of left action of invertible matrix having order of $2 \times 2$ on $G F\left(2^{8}\right)$ to generate elements of S-box. Moreover, to improve the confusion aptitude of erected S-box we exerted 1's and 2's compliment's technique for shuffling of an elements of S-box. Eventually, to inspect the capacity of designed S-box we bring into effective action of different procedures from literature such as strict avalanche criterion, nonlinearity, linear approximation probability, bit independence criterion and differential approximation probability.
\end{abstract}

Keywords: Invertible mapping, 1's compliment, 2's compliment, Cryptographic features. 


\section{Introduction}

Due to fast and latest developments in the field of information technology security of confidential information becomes very important. Different organizations and companies are needed the protection of their important information because the concealment of confidential data may be cause of collapse of whole organization or company. To overcome this type of problem a different encryption algorithms are introduced in literature such as Advanced Encryption Standard (AES) and Data Encryption Standard (DES) etc. AES and DES encryption algorithms utilized Substitution-box (S-box) to create confusion during the process of encryption so that an attacker cannot obtain confidential date easily due to confusion capability [1]. The recent symmetric cryptosystems relies on significant constituents known as S-box. This nonlinear constituent produces nonlinearity to increase confusion during encryption as well as increases the security for cryptosystems. Due to confusion creating ability S-box is most important component of AES algorithm and a number of cryptographers are showing their interests to improve confusion creating capability of S-box. In this research proposal, we propose to enhance the confusion creating capacity of S-box as well as increasing its security against some differential and linear attacks. First of all we generated 256 elements of $G F\left(2^{8}\right)$ utilizing a specific type of primitive polynomial [2]-[3]. Secondly we constructed a transformed S-box after utilization of left action of $2 \times 2$ invertible matrix on elements of Galois field $G F\left(2^{8}\right)$. To enhance the confusion ability of transformed S-box we applied two different techniques of 1's compliment and 2's compliment [4]. To obtain revised S-box we applied compliment methods and altered the elements with corresponding values which are generated using primitive polynomial. The proposed methodology for the construction of transformed S-box and revised Sbox is also graphically presented in Fig.1. Additionally, to observe the confusion ability and strength of transformed S-box and revised S-box we also critically analyzed these S-boxes for well-known cryptographic properties. In the end, we made comparison of transformed S-box and revised S-box with renowned $S$-boxes from literature such as Skipjack S-box [5], $S_{8}$ Liu J Sbox [6], Hussain [7] and Residue Prime S-box [8].
In this research paper we arranged the whole work as follows: in section 2 we briefly described a technique used to generate elements of $G F\left(2^{8}\right)$ and also discussed about stepwise procedure to design transformed and revised S-boxes. Furthermore, mathematical model used for the construction of both S-boxes is completely discussed in section 2. Section 3 includes the assessment of constructed S-boxes and their comparison with renowned S-boxes after utilization of important cryptographic properties. Section 4 deals with the conclusion of research article.

\section{Step-Wise Procedure and Mathematical Model for Proposed Method}

To design the substitution box for better encryption, we have designed following procedure,

Step 1: First of all we generate elements of $G F\left(2^{8}\right)$ through the utilization of specific primitive polynomial $p(\varphi)=1+\varphi^{2}+\varphi^{3}+$ $\varphi^{4}+\varphi^{8}$ which implies that $1+\varphi^{2}+\varphi^{3}+\varphi^{4}+$ $\varphi^{8}=0$. Then generated values of $\varphi$ under modulo $p(\varphi)$ are listed in Table 1 in terms of $\varphi$.

Step 2: An invertible mapping $y(x)=(m x+$ $n) /(r x+s)$ is designed after the application of left action of invertible matrix $\left(\begin{array}{cc}m & n \\ r & s\end{array}\right)$ on $G F\left(2^{8}\right)$, where $m . s-n \cdot r \neq 0$ and $m, n, r, s \in G F\left(2^{8}\right)$

Step 3: Since $m, n, r, s \in G F\left(2^{8}\right)$ then the values of $m, n, r, s$ are varying from 0:255 under a certain condition that $m . s-n . r \neq 0$. For particular instances $\mathrm{m}=35, \mathrm{n}=23, \mathrm{r}=14$ and $\mathrm{s}=9$ then we have $y(x)=(35 x+23) /(14 x+9)$.

Step 4: Utilization of particular primitive polynomial to find elements of transformed Sbox $y(0), y(1), y(2), \ldots, y(255)$.

Step 5: To enhance the confusion ability of Sbox we applied 1's compliment method.

Step 6: Lastly, we utilized 2's compliment technique to increase more confusion after the toggling of elements of S-box.

Step 7: In the end, elements are replaced with corresponding elements from Table 1 to get elements of revised S-box. 
As we already know that there exist a number of invertible mappings in the field of mathematics but for better encryption power we must need a powerful invertible mapping. For this purpose, we firstly construct an invertible mapping through the utilization of a left action of a projective linear group such as

$$
\begin{array}{r}
y:\left(\begin{array}{cc}
35 & 23 \\
14 & 9
\end{array}\right) \times G F\left(2^{8}\right) \rightarrow G F\left(2^{8}\right) \\
y(x)=\frac{35 x+23}{14 x+9}, \quad \forall x \in G F\left(2^{8}\right)
\end{array}
$$

The elements of transformed S-box are calculated using transformation presented in (1) and for these purpose the values of $x=0: 255$ are applied. The constructed elements of transformed S-box are indicated in $16 \times 16$ matrix presented in Table 2. Afterward, the elements of transformed S-box are converted into corresponding binary number system to enhance their confusion capability after utilization of 1 's and 2's compliment methods.

\subsection{Application of 1's Compliment Method}

i. At initial stage elements of transformed S-box are converted in 8-bits representation.

ii. Utilization of ' 0 ' as MSB to complete 8bits representation when numbers of bits are less than 8 .

iii. To find 1 's compliment of selected element of S-box in 8-bits form, a number is subtracted from the binary number which consists of same binary digits which are all equal to 1 .

\subsection{Application of 2's Compliment Method}

i. Before application of 2's compliment technique elements of S-box must be converted into 8-bits form after utilization of ' 0 ' as MSB.

ii. Apply 2's compliment method on elements obtained after application of 1 's compliment.

iii. To find 2's compliment of selected element of S-box in 8-bits form, flip all bits 0 into 1 and 1 into 0 from right side but without any change to the first 1 .

After the application of compliment's techniques the elements of revised S-box are presented in Table 3 for further comparison.

\section{Assessment of Transformed S-Box and Revised S-Box for Encryption Abilities} 3.1 Comparison of Nonlinearity Analysis Nonlinearity is most applicable property applied on S-boxes to analyze the confusion ability of Sboxes. The best value of nonlinearity for constructed S-boxes is equal to 120 for eight binary digits and this value can be calculated by using $N_{f}=\frac{2^{n}-2^{n / 2}}{2}=120$, where $n=$ number of bits [9]-[10]. Nonlinearity of revised S-box and other renowned S-boxes are calculated by utilization of analysis software [11].

Furthermore, analysis report of constructed Sboxes and $\mathrm{S}$-boxes from literature is presented in Table 4 for comparison of confusion creating capacity. Nonlinearity behavior of compared Sboxes is also interpreted in Figure 2. Analysis of transformed and revised S-boxes indicates that compliment technique increases confusion capability from 103.25 to 105.25 .

Also Table 4 and graphical interpretation of average nonlinearity represents that confusion capacity (105.25) of revised S-box is comparatively better than transformed S-box, Residue prime S-box (99.5), Husain's S-box (104.75) and $S_{8}$ Liu J S-box (104.875). Moreover, nonlinearity value is also very close to the value of Skipjack S-box (105.75).

Table 1: Generated Elements of $G F\left(2^{8}\right)$ Corresponding to Polynomial $\left[\begin{array}{llllllll}1 & 0 & 1 & 1 & 1 & 0 & 0 & 0\end{array}\right]$

\begin{tabular}{cccc}
\hline $\mathbf{G F}\left(2^{8}\right.$ & Binary & $\mathbf{G F}\left(2^{8}\right.$ & Binary \\
) & Form & ) & Form \\
\hline 0 & 00000000 & $\varphi^{7}$ & 00100110 \\
$\varphi$ & 00010110 & $\varphi^{8}$ & 01000101 \\
$\varphi^{2}$ & 00110111 & $\varphi^{9}$ & 01100010 \\
$\varphi^{3}$ & 00010010 & $\cdot$ & $\cdot$ \\
$\varphi^{4}$ & 00001000 & $\cdot$ & $\cdot$ \\
$\varphi^{5}$ & 00110100 & $\varphi^{254}$ & 10001110 \\
$\varphi^{6}$ & 01000000 & $\varphi^{255}$ & 00000001 \\
\hline
\end{tabular}




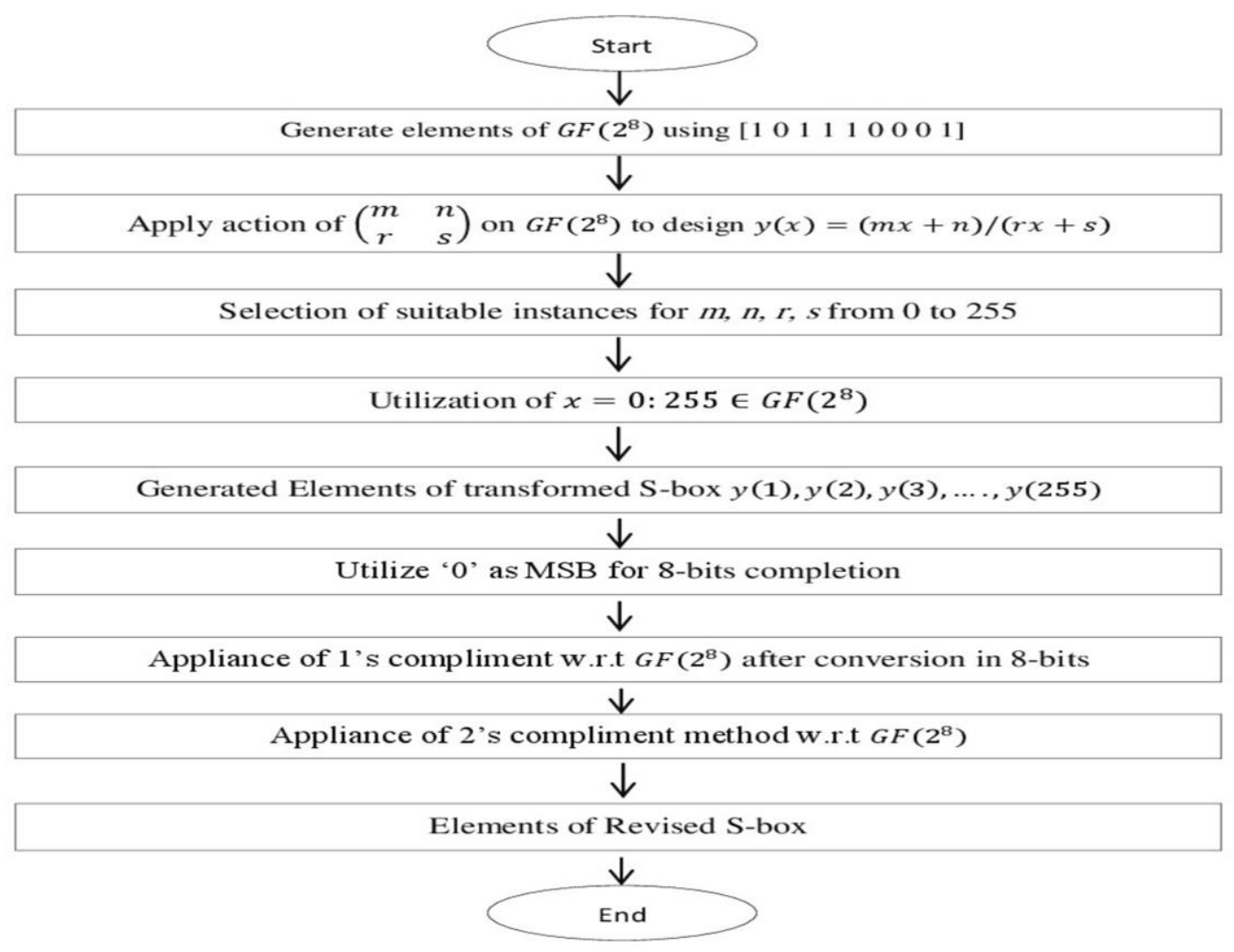

Fig. 1: Graphical overview of proposed scheme

3.2 Strict Avalanche Criterion (SAC) of Revised S-Box and Their Comparison Report Davida and Kam [12] suggested the notion of completeness and moreover Feistel [13] proposed a concept of avalanche effect. A transformation will satisfy the concept of completeness if every bit of ciphertext depends on bits of plaintext. Additionally, transformation satisfies the condition of avalanche effect if $50 \%$ output binary digits are going to be changed due to change in a single input bit. According to definition an S-box must satisfy the condition of $\mathrm{SAC}$ if average value is equal to 0.5 [14].
Calculated minimum, maximum and average values of SAC of revised S-box and other Sboxes are presented in Table 5 for comparison. Comparison detail is also graphically indicated in Fig. 3 which shows that the average value of $\mathrm{SAC}$ of revised S-box is approximately close to 0.5 and comparatively better than all other $\mathrm{S}$ boxes picked from literature for comparison.

\subsection{Assessment of Bits Independence Criterion (BIC)}

Bits independence criterion is well-known and desirable property was firstly introduced by 
Table 2: Elements of Transformed S-Box Produced by Invertible Function

\begin{tabular}{|c|c|c|c|c|c|c|c|c|c|c|c|c|c|c|c|c|}
\hline & 0 & 1 & 2 & 3 & 4 & 5 & 6 & 7 & 8 & 9 & 10 & 11 & 12 & 13 & 14 & 15 \\
\hline 0 & 237 & 57 & 180 & 90 & 61 & 146 & 120 & 154 & 219 & 22 & 91 & 201 & 232 & 132 & 137 & 134 \\
\hline 1 & 32 & 229 & 160 & 50 & 19 & 119 & 114 & 117 & 14 & 194 & 71 & 161 & 190 & 105 & 131 & 97 \\
\hline 2 & 60 & 0 & 30 & 149 & 40 & 55 & 83 & 203 & 121 & 252 & 222 & 80 & 216 & 228 & 199 & 213 \\
\hline 3 & 130 & 254 & 29 & 171 & 35 & 23 & 52 & 38 & 191 & 253 & 221 & 102 & 211 & 196 & 168 & 48 \\
\hline 4 & 107 & 205 & 244 & 206 & 82 & 78 & 21 & 212 & 217 & 158 & 178 & 138 & 110 & 238 & 16 & 63 \\
\hline 5 & 34 & 93 & 73 & 113 & 141 & 13 & 235 & 188 & 144 & 46 & 7 & 45 & 169 & 173 & 230 & 39 \\
\hline 6 & 234 & 18 & 4 & 118 & 163 & 224 & 187 & 231 & 197 & 170 & 226 & 142 & 248 & 126 & 200 & 68 \\
\hline 7 & 54 & 81 & 25 & 109 & 3 & 125 & 51 & 183 & 17 & 233 & 247 & 133 & 88 & 27 & 64 & 20 \\
\hline 8 & 1 & 176 & 6 & 150 & 77 & 76 & 41 & 140 & 47 & 155 & 5 & 193 & 208 & 198 & 192 & 95 \\
\hline 9 & 204 & 96 & 246 & 58 & 43 & 53 & 59 & 156 & 250 & 75 & 245 & 101 & 174 & 175 & 210 & 111 \\
\hline 10 & 42 & 243 & 28 & 44 & 94 & 103 & 220 & 215 & 162 & 129 & 116 & 67 & 11 & 9 & 153 & 72 \\
\hline 11 & 89 & 122 & 135 & 184 & 240 & 242 & 98 & 179 & 209 & 8 & 223 & 207 & 157 & 148 & 31 & 36 \\
\hline 12 & 128 & 85 & 195 & 104 & 87 & 241 & 159 & 70 & 69 & 185 & 255 & 62 & 139 & 145 & 236 & 124 \\
\hline 13 & 182 & 66 & 251 & 189 & 112 & 106 & 15 & 127 & 123 & 166 & 56 & 147 & 164 & 10 & 92 & 181 \\
\hline 14 & 202 & 33 & 37 & 186 & 172 & 249 & 26 & 100 & 115 & 86 & 152 & 12 & 108 & 84 & 74 & 24 \\
\hline 15 & 136 & 165 & 99 & 239 & 143 & 167 & 177 & 227 & 49 & 218 & 214 & 65 & 79 & 2 & 225 & 151 \\
\hline
\end{tabular}

Table 3: Revised S-Box Erected By Application of Compliment Method

\begin{tabular}{|c|cccccccccccccccc|}
\hline \multicolumn{1}{|c}{$\mathbf{0}$} & $\mathbf{1}$ & $\mathbf{2}$ & $\mathbf{3}$ & $\mathbf{4}$ & $\mathbf{5}$ & $\mathbf{6}$ & $\mathbf{7}$ & $\mathbf{8}$ & $\mathbf{9}$ & $\mathbf{1 0}$ & $\mathbf{1 1}$ & $\mathbf{1 2}$ & $\mathbf{1 3}$ & $\mathbf{1 4}$ & $\mathbf{1 5}$ \\
\hline $\mathbf{0}$ & $\mathbf{2 3 8}$ & $\mathbf{2 0 6}$ & $\mathbf{2 2 4}$ & $\mathbf{1 3 6}$ & $\mathbf{2 5}$ & $\mathbf{1 4 0}$ & $\mathbf{1 0 7}$ & $\mathbf{1 9 0}$ & $\mathbf{1 7 6}$ & $\mathbf{1 9 5}$ & $\mathbf{1 5 4}$ & $\mathbf{1 7 1}$ & $\mathbf{7 9}$ & $\mathbf{4 9}$ & $\mathbf{2 1 0}$ & $\mathbf{1 3 8}$ \\
$\mathbf{1}$ & $\mathbf{1 0 4}$ & $\mathbf{1 4 1}$ & $\mathbf{3 6}$ & $\mathbf{2 1 2}$ & $\mathbf{1 0 8}$ & $\mathbf{1 9 1}$ & $\mathbf{2 8}$ & $\mathbf{1 1 6}$ & $\mathbf{5 1}$ & $\mathbf{2 4 1}$ & $\mathbf{2 3 7}$ & $\mathbf{1 9}$ & $\mathbf{2 2 7}$ & $\mathbf{1 3 7}$ & $\mathbf{1 2 8}$ & $\mathbf{4 0}$ \\
$\mathbf{2}$ & $\mathbf{2 2 3}$ & $\mathbf{1 6 3}$ & $\mathbf{1 1 7}$ & $\mathbf{2 4 6}$ & $\mathbf{8}$ & $\mathbf{1 6 2}$ & $\mathbf{3 0}$ & $\mathbf{5 9}$ & $\mathbf{9 1}$ & $\mathbf{2 1 8}$ & $\mathbf{2 3 9}$ & $\mathbf{2 2 0}$ & $\mathbf{1 6 6}$ & $\mathbf{9 3}$ & $\mathbf{6 5}$ & $\mathbf{1 6 7}$ \\
$\mathbf{3}$ & $\mathbf{2 5 5}$ & $\mathbf{4 7}$ & $\mathbf{2 1 1}$ & $\mathbf{1 6}$ & $\mathbf{1 1 9}$ & $\mathbf{5 4}$ & $\mathbf{1 8 3}$ & $\mathbf{1 4 7}$ & $\mathbf{2 2 6}$ & $\mathbf{2 1 3}$ & $\mathbf{1 7}$ & $\mathbf{1 2}$ & $\mathbf{8 0}$ & $\mathbf{5}$ & $\mathbf{1 4 5}$ & $\mathbf{1 0 3}$ \\
$\mathbf{4}$ & $\mathbf{5 8}$ & $\mathbf{1 8 1}$ & $\mathbf{2 3 4}$ & $\mathbf{1 2 4}$ & $\mathbf{4 1}$ & $\mathbf{2 0 8}$ & $\mathbf{1 0 1}$ & $\mathbf{1 4 9}$ & $\mathbf{9 6}$ & $\mathbf{7 6}$ & $\mathbf{1}$ & $\mathbf{1 3}$ & $\mathbf{1 2 0}$ & $\mathbf{1 0}$ & $\mathbf{1 8 5}$ & $\mathbf{8 7}$ \\
$\mathbf{5}$ & $\mathbf{2 0 0}$ & $\mathbf{5 0}$ & $\mathbf{2 4 5}$ & $\mathbf{2 3 2}$ & $\mathbf{2 4 0}$ & $\mathbf{1 6 8}$ & $\mathbf{1 3 0}$ & $\mathbf{1 1 5}$ & $\mathbf{2 4}$ & $\mathbf{1 8 6}$ & $\mathbf{1 5 2}$ & $\mathbf{1 5 8}$ & $\mathbf{1 0 0}$ & $\mathbf{7 2}$ & $\mathbf{2 1}$ & $\mathbf{2 4 3}$ \\
$\mathbf{6}$ & $\mathbf{3}$ & $\mathbf{2 3}$ & $\mathbf{1 6 1}$ & $\mathbf{1 7 9}$ & $\mathbf{1 7 7}$ & $\mathbf{2 0 2}$ & $\mathbf{6 4}$ & $\mathbf{1 5 6}$ & $\mathbf{4 2}$ & $\mathbf{1 5}$ & $\mathbf{1 9 6}$ & $\mathbf{1 3 3}$ & $\mathbf{1 3 2}$ & $\mathbf{1 4 6}$ & $\mathbf{3 3}$ & $\mathbf{1 1 2}$ \\
$\mathbf{7}$ & $\mathbf{1 7 5}$ & $\mathbf{2 2 8}$ & $\mathbf{1 2 6}$ & $\mathbf{2 2 2}$ & $\mathbf{5 7}$ & $\mathbf{2 1 5}$ & $\mathbf{1 8 4}$ & $\mathbf{1 1 4}$ & $\mathbf{6 6}$ & $\mathbf{2 1 6}$ & $\mathbf{2 4 9}$ & $\mathbf{2 3 0}$ & $\mathbf{9}$ & $\mathbf{1 5 7}$ & $\mathbf{1 1 0}$ & $\mathbf{2 2 1}$ \\
$\mathbf{8}$ & $\mathbf{9 9}$ & $\mathbf{9 8}$ & $\mathbf{1 6 4}$ & $\mathbf{4 8}$ & $\mathbf{2 5 0}$ & $\mathbf{7 8}$ & $\mathbf{2 3 6}$ & $\mathbf{6 1}$ & $\mathbf{6 2}$ & $\mathbf{8 6}$ & $\mathbf{3 8}$ & $\mathbf{8 6}$ & $\mathbf{5 5}$ & $\mathbf{1 3 4}$ & $\mathbf{1 7 0}$ & $\mathbf{1 8 0}$ \\
$\mathbf{9}$ & $\mathbf{2 0 9}$ & $\mathbf{2 1 9}$ & $\mathbf{6 3}$ & $\mathbf{2 2 5}$ & $\mathbf{1 2 2}$ & $\mathbf{2 3 5}$ & $\mathbf{1 1 3}$ & $\mathbf{2 6}$ & $\mathbf{1 9 2}$ & $\mathbf{1 4 2}$ & $\mathbf{1 2 5}$ & $\mathbf{9 2}$ & $\mathbf{9 4}$ & $\mathbf{2 0 3}$ & $\mathbf{1 4 4}$ & $\mathbf{2 5 1}$ \\
$\mathbf{1 0}$ & $\mathbf{1 2 7}$ & $\mathbf{1 5 1}$ & $\mathbf{4}$ & $\mathbf{8 8}$ & $\mathbf{6 0}$ & $\mathbf{4 3}$ & $\mathbf{6 8}$ & $\mathbf{1 2 3}$ & $\mathbf{1 7 8}$ & $\mathbf{1 9 4}$ & $\mathbf{2 0 1}$ & $\mathbf{2 3 1}$ & $\mathbf{5 2}$ & $\mathbf{5 3}$ & $\mathbf{1 8}$ & $\mathbf{1 9 8}$ \\
$\mathbf{1 1}$ & $\mathbf{1 5 0}$ & $\mathbf{1 0 2}$ & $\mathbf{2 1 4}$ & $\mathbf{1 0 9}$ & $\mathbf{9 5}$ & $\mathbf{2 2 9}$ & $\mathbf{1 6 5}$ & $\mathbf{1 9 7}$ & $\mathbf{1 0 6}$ & $\mathbf{1 1}$ & $\mathbf{2 0 7}$ & $\mathbf{1 7 2}$ & $\mathbf{3 2}$ & $\mathbf{1 1 8}$ & $\mathbf{1 2 9}$ & $\mathbf{7 7}$ \\
$\mathbf{1 2}$ & $\mathbf{1 7 4}$ & $\mathbf{2 4 2}$ & $\mathbf{2 5 2}$ & $\mathbf{8 1}$ & $\mathbf{8 5}$ & $\mathbf{2 3 3}$ & $\mathbf{2 0}$ & $\mathbf{1 3 5}$ & $\mathbf{2 4 8}$ & $\mathbf{7 1}$ & $\mathbf{1 9 9}$ & $\mathbf{1 0 5}$ & $\mathbf{6}$ & $\mathbf{4 5}$ & $\mathbf{2 5 3}$ & $\mathbf{0}$ \\
$\mathbf{1 3}$ & $\mathbf{9 7}$ & $\mathbf{9 0}$ & $\mathbf{2 0 5}$ & $\mathbf{1 9 3}$ & $\mathbf{6 9}$ & $\mathbf{1 8 9}$ & $\mathbf{1 3 1}$ & $\mathbf{2}$ & $\mathbf{7}$ & $\mathbf{8 4}$ & $\mathbf{3 5}$ & $\mathbf{2 1 7}$ & $\mathbf{1 8 2}$ & $\mathbf{1 6 0}$ & $\mathbf{2 9}$ & $\mathbf{1 6 9}$ \\
$\mathbf{1 4}$ & $\mathbf{2 4 7}$ & $\mathbf{5 6}$ & $\mathbf{7 0}$ & $\mathbf{1 5 5}$ & $\mathbf{1 5 3}$ & $\mathbf{3 4}$ & $\mathbf{1 4 3}$ & $\mathbf{1 8 7}$ & $\mathbf{6 7}$ & $\mathbf{4 4}$ & $\mathbf{1 4}$ & $\mathbf{1 1 1}$ & $\mathbf{7 4}$ & $\mathbf{1 2 1}$ & $\mathbf{2 0 4}$ & $\mathbf{8 3}$ \\
15 & $\mathbf{3 7}$ & $\mathbf{1 5 9}$ & $\mathbf{3 1}$ & $\mathbf{2 2}$ & $\mathbf{7 5}$ & $\mathbf{8 9}$ & $\mathbf{4 6}$ & $\mathbf{1 4 8}$ & $\mathbf{2 4 4}$ & $\mathbf{1 7 3}$ & $\mathbf{2 7}$ & $\mathbf{1 8 8}$ & $\mathbf{7 3}$ & $\mathbf{2 5 4}$ & $\mathbf{1 3 9}$ & $\mathbf{3 9}$ \\
\hline
\end{tabular}


Table 4: Nonlinearity Comparison of Revised S-Box with Renowned S-Boxes

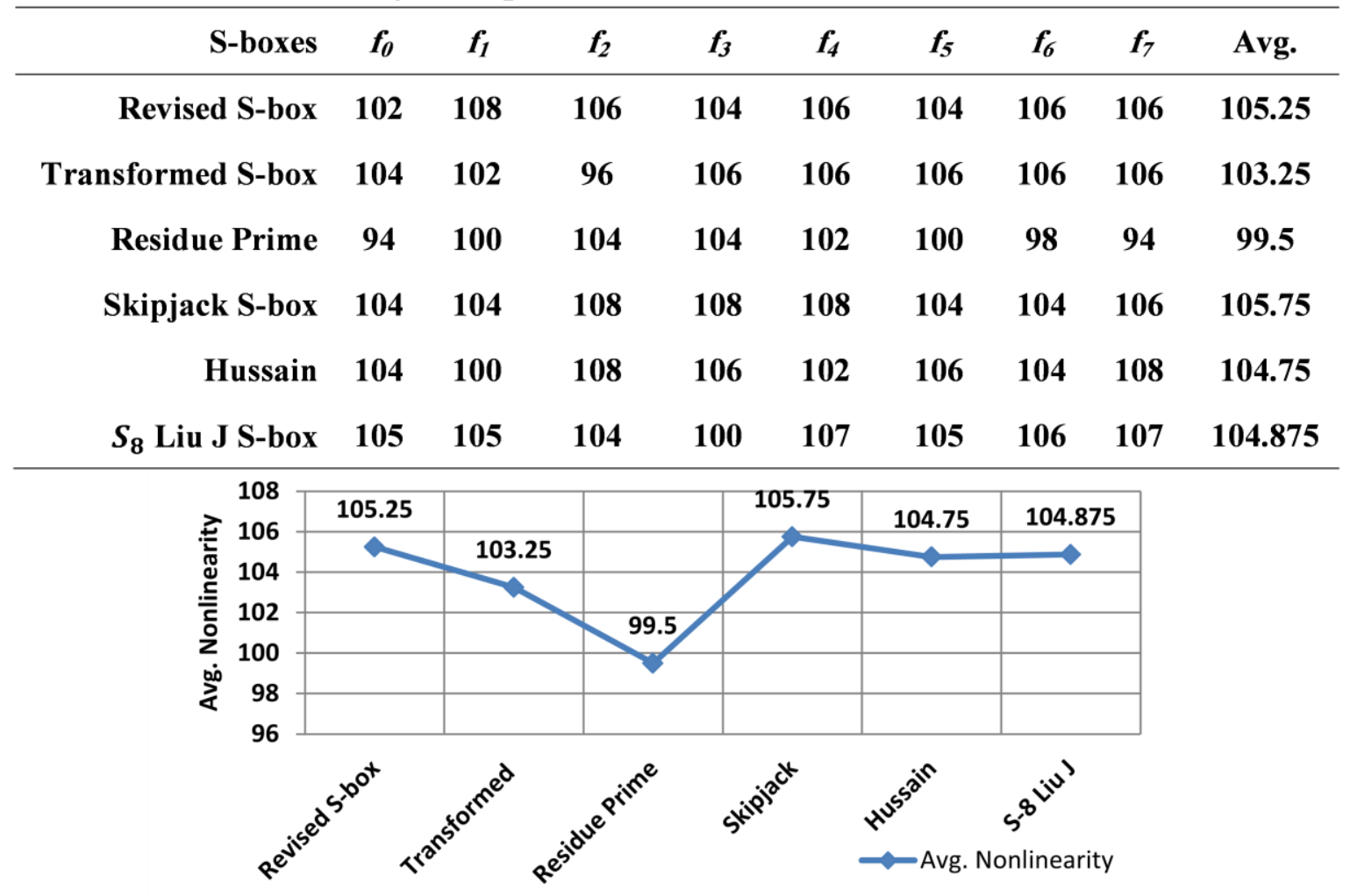

Fig. 2: Average Nonlinearitv comnarison

Table 5: Assessment of SAC of Revised S-Box and Their Comparison

\begin{tabular}{rccc}
\hline S-boxes & Avg. value & Min. value & Max. value \\
\hline Revised S-box & $\mathbf{0 . 5 0 2 1 9 7}$ & $\mathbf{0 . 4 0 6 2 5}$ & $\mathbf{0 . 6 2 5}$ \\
Transformed S-box & $\mathbf{0 . 4 9 2 4 3 2}$ & $\mathbf{0 . 4 0 6 2 5}$ & $\mathbf{0 . 5 9 3 7 5}$ \\
Residue Prime & 0.51 & 0.343 & 0.67 \\
Skipjack S-box & 0.53 & 0.39 & 0.59 \\
Hussain & 0.49 & 0.391 & 0.59 \\
$S_{\mathbf{8}}$ Liu J S-box & 0.499 & 0.429 & 0.59 \\
\hline
\end{tabular}

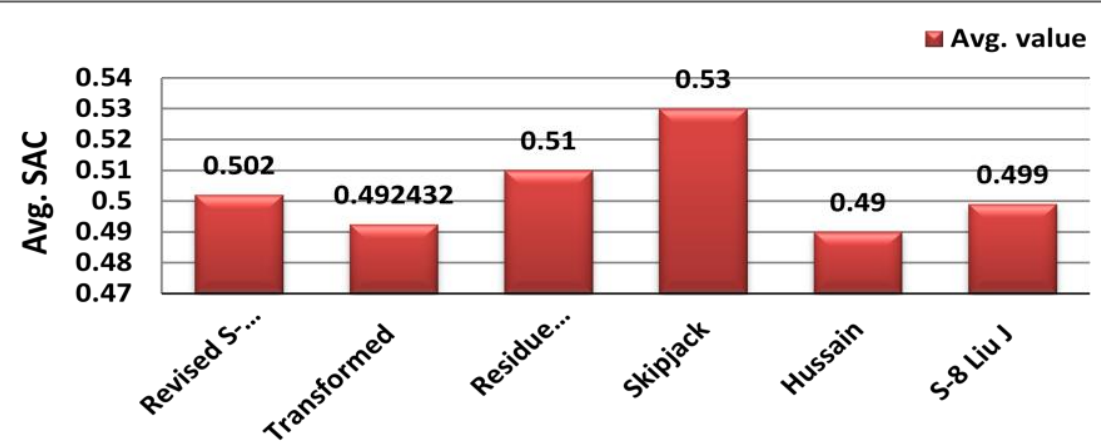

Fig. 3: Analysis comparison of average value of SAC 
Tavares and Webster [15]. This property analyzes the change of output binary digits when input binary digits of plaintext are complemented. Also we observe the independent of two output bits when one input bit is altered. The test of BIC is applied on nonlinearity of revised S-box, transformed S-box, residue prime, skipjack, Husain's S-box and $\mathrm{S}_{8^{-}}$ Liu J S-boxes.

Table 6: Comparison of BIC of Nonlinearity for Renowned S-Boxes with Revised S-Box

\begin{tabular}{lcc}
\hline S-boxes & Avg. value & Min. value \\
\hline Revised S-box & 103.643 & 96 \\
Transformed S-box & 100.429 & 92 \\
Residue Prime & 101.71 & 94 \\
Skipjack S-box & 104.14 & 102 \\
Hussain & 105.071 & 100 \\
$S_{8}$ Liu J S-box & 104.786 & 99 \\
\hline
\end{tabular}

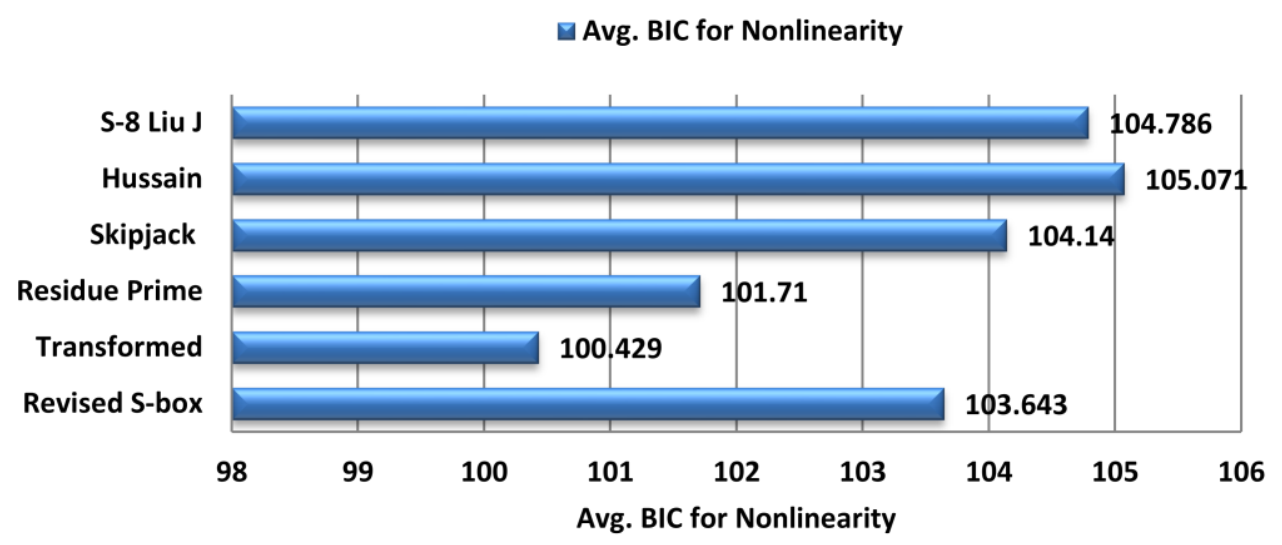

Fig. 4: Graphical Interpretation of BIC for Nonlinearity

\subsection{Analysis of Differential Approximation Probability (DAP)}

The analysis of differential approximation probability (DAP) is also most important property to observe the strength of S-boxes against some differential attacks. The differential probability value specifies the resistance of S-box against differential attacks. According to DAP property input differential $\Delta x$ must uniquely map to $\Delta y$ at output level to calculate differential pair $(\Delta x, \Delta y)$ such that:

Input differential: $\Delta x$

Output differential: $\Delta \mathrm{y}=\mathrm{S}(\mathrm{x}) \oplus \mathrm{S}(\mathrm{x} \oplus \Delta \mathrm{x})$
Mathematically DAP is defined in [16] for 8-bits as follows,

$$
\mathrm{DP}_{(\Delta \mathrm{x} \rightarrow \Delta \mathrm{y})}=\left[\frac{\#\{\mathrm{x} \in \mathrm{X} / \mathrm{S}(\mathrm{x}) \oplus \mathrm{S}(\mathrm{x} \oplus \Delta \mathrm{x})=\Delta \mathrm{y}\}}{256}\right]
$$

The proposed revised S-box and other S-boxes are analyzed for DAP test and results are described in Table 7. The minimum DAP value of S-box indicates that there are less chances of attacks. Graphical behavior of DAP from Fig. 5 shows that the probability value of revised S-box is superior to all other S-boxes except $S_{8}$ Liu J Sbox because both have same probability value. 
Table 7: Analysis Report of Differential Approximation Probability

\begin{tabular}{ccccccc}
\hline S-boxes & Revised S-box & $\begin{array}{c}\text { Transformed } \\
\text { S-box }\end{array}$ & $\begin{array}{c}\text { Residue } \\
\text { Prime }\end{array}$ & Skipjack & Hussain & $\begin{array}{c}S_{8} \text { Liu J } \\
\text { S-box }\end{array}$ \\
\hline Max. DAP & $\mathbf{0 . 0 3 9 0 6 2 5}$ & $\mathbf{0 . 4 9 2 1 8 8}$ & $\mathbf{0 . 2 8 1}$ & $\mathbf{0 . 0 4 6 8}$ & $\mathbf{0 . 1 2 5}$ & $\mathbf{0 . 0 3 9 0}$ \\
\hline
\end{tabular}

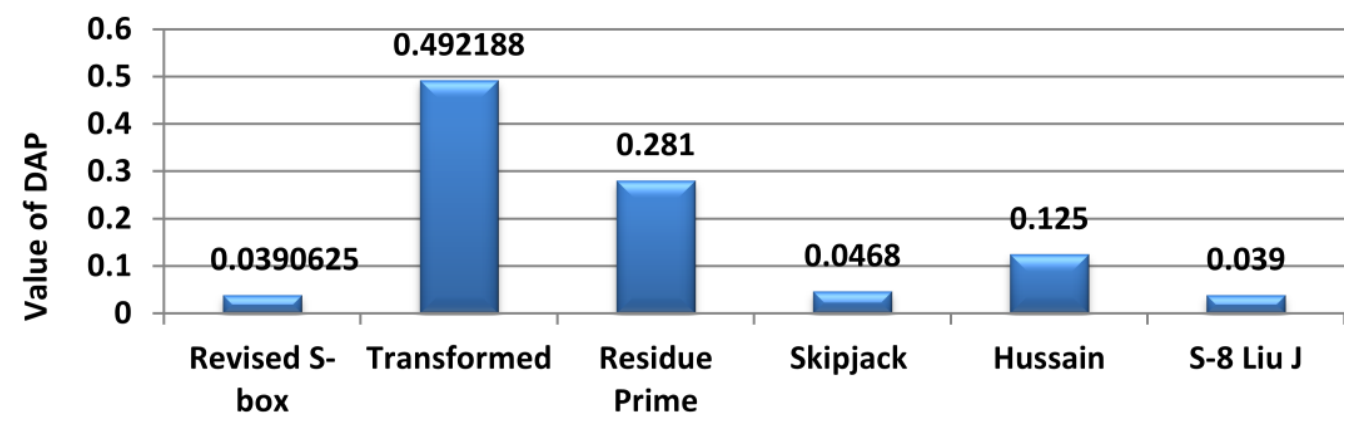

Fig. 5: Graphical performance of Maximum DAP

3.5 Behavior of S-Box against Linear Attacks

The linear approximation probability (LAP) is most important cryptographic property which is utilized to analyze the imbalance of an event. The LAP value represents the resistance of S-box against linear attacks. The value of LAP come close to probability of zero is considered as best value because it reduces the chances of linear attacks on ciphertext. The best supreme value of LAP is 0 because it indicates zero chances of attacks but only in rare cases it could happen. For input bits the mask $\Gamma m$ and for output bits the mask $\Gamma n$ are utilized. The definition of LAP for 8-bits is described in [17] as follows,

$$
L P=\underset{\Gamma m, \Gamma n \neq 0}{\operatorname{Max}} \mid \frac{\text { Number } \text { of }\{x \in X / x . \Gamma m=S(x) . \Gamma n\}}{256}
$$

Analysis results of S-boxes are listed in Table 8 to make comparison. Maximum value of LP for all S-boxes are graphically interpreted in Fig. 6 and comparison shows that after application of compliment technique the LP value of revised S-box $(0.132813)$ is better than transformed S-box (0.148438). Also resistance of revised $\mathrm{S}$-box against linear attacks is identical to residue prime $\mathrm{S}$-box and comparable with other S-boxes.

Table 8: Analysis and Comparison of Linear Approximation Probability

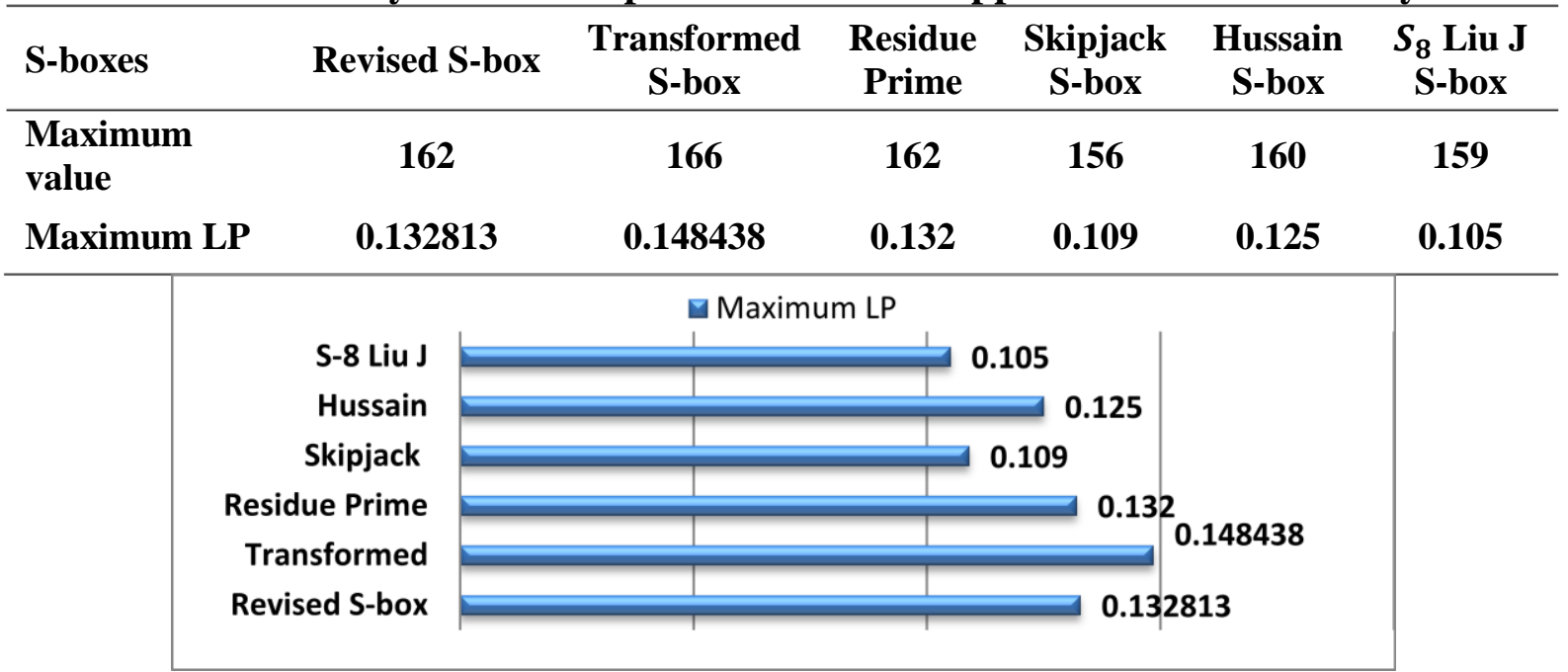

Fig. 6: Graphical presentation of maximum LP 


\section{Conclusion}

This research proposal originated an innovative mechanism for the erection of Substitution box (S-box) with the assistance of implementation of invertible function. To acquire high encryption strength and confusion ability we employed 1's and 2's compliment technique on transformed S-box and derived Revised S-box. To inspect the information encryption capacity of Revised S-box, we perform comparison with eminent S-boxes from literature. We implement comparison of S-boxes through well-known cryptographic properties and investigation report indicates that fabrication of Revised S-box is superior to some S-boxes. The analysis behavior of Revised S-box represents that 1's and 2's compliment methodology is very reliable to increase encryption capabilities of S-boxes. Furthermore, property of differential probability specifies that Revised S-box can create greater resistance against differential attacks when compared with other S-boxes. Therefore, Revised S-box can be utilized in any encryption algorithm to protect confidential information.

\section{Acknowledgement}

This work was supported by the National Natural Science Foundation of China (11571378). We are thankful to the editor and unknown reviewers for their valuable suggestions which have improved the quality of the paper to a large extent.

\section{References}

[1] Daemen, Joan, and Vincent Rijmen. The design of Rijndael: AES-the advanced encryption standard. Springer Science \& Business Media, 2013.

[2] Hussain, Iqtadar, Tariq Shah, Hasan Mahmood, and Muhammad Asif Gondal. "A projective general linear group based algorithm for the construction of substitution box for block ciphers." Neural Computing and Applications 22, no. 6 (2013): 1085-1093.

[3] Sarfraz Muhammad, Iqtadar Hussain, and Fateh Ali. "Construction of S-Box Based on Mobius Transformation and Increasing Its Confusion Creating Ability through Invertible Function." International Journal of Computer Science and Information Security 14, no. 2 (2016): 187.

[4] Parhami, Behrooz. Computer arithmetic. Vol. 20, no. 00. Oxford university press, 1999.

[5] KIM, J. and PHAN, R.C.-W., 2009. Advanced differential-style cryptanalysis of the NSA's skipjack block cipher. Cryptologia, 33(3), pp. 246-270.

[6] Hussain, Iqtadar, Tariq Shah, Hasan Mahmood, and Muhammad Asif Gondal. "Construction of S 8 Liu J S-boxes and their applications." Computers \& Mathematics with Applications 64, no. 8 (2012): 2450-2458.

[7] Hussain, Iqtadar, Tariq Shah, Muhammad Asif Gondal, Waqar Ahmad Khan, and Hasan Mahmood. "A group theoretic approach to construct cryptographically strong substitution boxes." Neural Computing and Applications 23, no. 1 (2013): 97-104.

[8] Hussain, Iqtadar, Tariq Shah, Hasan Mahmood, Muhammad Asif Gondal, and Usman Younas Bhatti. "Some analysis of S-box based on residue of prime number." Proc Pak Acad Sci 48, no. 2 (2011): 111-115. 
[9] Meier, Willi, and Othmar Staffelbach. "Nonlinearity criteria for cryptographic functions." In Workshop on the Theory and Application of of Cryptographic Techniques, pp. 549-562. Springer, Berlin, Heidelberg, 1989.

[10]Hussain, Iqtadar, Tariq Shah, Hasan Mahmood, and Muhammad Asif Gondal. "Construction of S 8 Liu J S-boxes and their applications." Computers \& Mathematics with Applications 64, no. 8 (2012): 2450-2458.

[11]Wang, Yong, Qing Xie, Yuntao Wu, and Bing Du. "A software for S-box performance analysis and test." In Electronic commerce and business intelligence, 2009. ECBI 2009. International Conference on, pp. 125-128. IEEE, 2009.

[12] Kam, John B., and George I. Davida. "Structured design of substitutionpermutation encryption networks." IEEE Transactions on Computers 10 (1979): 747-753.
[13] Feistel, Horst. "Cryptography and computer privacy." Scientific american 228, no. 5 (1973): 15-23.

[14] Webster AF, Tavares SE. On the design of S-boxes. In Conference on the Theory and Application of Cryptographic Techniques 1985 Aug 18 (pp. 523-534). Springer, Berlin, Heidelberg.

[15]Detombe, John, and Stafford Tavares. "Constructing large cryptographically strong S-boxes." In International Workshop on the Theory and Application of Cryptographic Techniques, pp. 165-181. Springer, Berlin, Heidelberg, 1992.

[16]Biham, Eli, and Adi Shamir. Differential cryptanalysis of the data encryption standard. Springer Science \& Business Media, 2012.

[17] Matsui, Mitsuru. "Linear cryptanalysis method for DES cipher." In Workshop on the Theory and Application of Cryptographic Techniques, pp. 386-397. Springer, Berlin, Heidelberg, 1993. 